\title{
Über Phosphoroxysulfid.
}

\author{
Von \\ T. E. Thorpe und A. E. Tutton. ${ }^{1}$
}

Erhitzt man Phosphorigsäureanhydrid und Schwefel in einer Atmosphäre von Kohlendioxyd oder Stickstoff, so schmelzen die beiden Substanzen zunächst und bilden getrenute Flüssigkeitsschichten. Bei ungeführ $160^{\circ}$ jedoch tritt eine heftige Reaction ein, und die Mischung wird fest; eine Gasentwickelung findet bei dieser Reaktion nicht statt. Die feste Substanz ist ein Additionsprodukt, das, wie wir nachweisen werden, die Formel $\mathrm{P}_{4} \mathrm{O}_{6} \mathrm{~S}_{4}$ besitzt. Direkte Versuche haben gezeigt, dals dasselbe quantitativ entsteht nach der Gleichung:

$$
\mathrm{P}_{4} \mathrm{O}_{6}+4 \mathrm{~S}=\mathrm{P}_{4} \mathrm{O}_{6} \mathrm{~S}_{4} \text {. }
$$

Es kann in grofsen, schön geformten Krystallen gewonnen werden durch Sublimation im luftleeren Raum, oder durch Krystallisation aus Schwefelkohlenstoff. Wir schlagen vor, die Substanz Phosphoroxysulfid zu nennen.

Phosphoroxysulfid kann leicht auf folgende Weise erhalten werden :

3 bis 5 Gramm frisch destilliertes Phosphortrioxyd, das nach der von uns beschriebenen Methode dargestellt ist, ${ }^{2}$ werden auf den Boden einer schwer schmelzbaren Glasröhre gebracht, die an einem Ende geschlossen und zuvor mit trockenem Kohlendioxyd, oder Stickstoff angefüllt ist. Die erforderliche Menge von Schwefel, wie sich dieselbe nach obiger Gleichung berechnet, wird dann am besten in Form von kleinen Krystallen hinzugefügt, die Röhre über dem Gebläse zugeschmolzen und mit der unteren Hälfte in ein Glycerinbad getaucht, dessen Temperatur allmählich erhöht wird. Bis zu $150^{\circ}$ ist keine Reaktion sichtbar, da der Schwefel nur bei $115^{0}$ schmilzt und eine Schichte unter dem Phosphorigsäureanhydrid bildet. Bei einer Temperatur, welche bei verschiedenen Versuchen zwischen $154^{\circ}$ und $168^{\circ}$ schwankte, wurde die untere Schwefelschichte plötzlich in das Phosphorigsäureanhydrid geschleudert und die ganze Masse mit einem deutlichen, zischenden Ton heftig bis an die Spitze der Röhre geworfen. Es ist wichtig, dafs die angewandten Quanti-

${ }^{1}$ Nach dem Manuskripte der Verfasser deutsch von (†. KRüss.

2 , Journ. chem. Soc., Transactions (1890) 553. 
täten die angegebenen Mengen nicht übersteigen, denn, wenn mehr als 5 Gramm Phosphorigsäureanhydrid und die entsprechende Menge von Schwefel angewendet werden, ist die Reaktion so heftig, daf sie gewöhnlich eine laute Explosion zur Folge hat und die Röhre in Stücke zerbricht. Eine intensiy helle Flamme begleitet die Explosion.

Werden die oben angegebenen Gewichtsmengen angewandt, so ist die Reaktion in zwei oder drei Sekunden beendet, während welcher Zeit sich der Inhalt der Röhre in lebhafter Bewegung befindet. Die innere Wand der Röhre bedeckt sich an den kühlleren Stellen mit gefiederten, farblosen Krystallen und zugleich mit kompakten Massen eines gelblich grauen, krystallinisch festen Körpers, der an den stärker erhitzten Teilen der Röhre zn einer zähen Flüssigkeit schmilzt. Das Erhitzen kann natürlich auch in einem Luftbad ausgeführt werden, aber es ist dann unmöglich die merkwürdigen Erscheinungen zu beobachten, welche die Reaktion begleiten. Das Produkt wird dann in eine ähnliche Röhre umgefüllt, aus dieser hierauf mit einer Sirengelschen Pumpe die Luft ausgepumpt und die Röhre zugeschmolzen. Erhitzt man die untere Hälfte der luftleeren Röhre, so sublimiert das Oxysulfid in vollständig farblosen, stark lichtbrechenden Krystallen.

Die Sublimation beginnt bei ungefähr $90^{\circ}$, die günstigste Temperatur ist jedoch bei ungefïhr $140^{\circ}-150^{\circ}$. Ein Teil des Sublimates verdichtet sich zuerst zn einer zähen Flüssigkeit, welche später zu einer farblos glasigen, oder krystallinischen Masse erstart; ein grofer Teil verdichtet sich jedoch immer in Form von gefiederten Aggregaten - lange Nadeln, die sich quer du'ch das Rohr erstrecken oder in isolierten, rektangulären Krystallen. Die Analyse hat gezeigt, dals diese verschiedenen Formen des Sublimates die gleiche Zusammensetzung besitzen.

Phosphoroxysulfid schmilzt bei ungefähr $102^{\circ}$ und siedet beständig bei $295^{\circ}$ (corr.). Der Schmelzpunkt ist nicht ganz scharf, da die Substanz etwas zähe zu werden scheint, bevor sie wirklich flüssig wird. Das destillierte Oxysulfid besitzt eine blafsgelbe Farbe und sublimiert beinahe vollständig im Vacuum in der Form der oben beschriebenen farblosen Krystalle.

Bei der Analyse durch Oxydation mittelst Bromwasser, bei der Bestimmung der Phosphorsäure mit einer Magnesiamischung und der Schwefelsäure mit Baryumchlorid lieferte es die folgenden Resultate, welche für die drei verschiedenen Präparate erhalten wurden: 


$\begin{array}{ccccc} & & & & \text { Berechnet für } \\ & \text { I. } & \text { II. } & \text { III. } & \mathrm{P}_{4} \mathrm{O}_{6} \mathrm{~S}_{4} \\ \text { Phosphor } & 35.81 & 35.71 & 35.94 & 35.63 \\ \text { Schwefel } & 36.08 & 36.88 & 36.36 & 36.78\end{array}$

Wir vermuteten zuerst, die Substanz könnte ein gemischtes Anhydrid sein, das heifst ein Phosphorsäureanhydrid, in welchem ein Teil des Sauerstoffs durch eine äquivalente Menge Schwefel ersetzt ist, oder $\mathrm{P}_{2} \mathrm{O}_{3} \mathrm{~S}_{2}$. Dafs die Substanz in der That die Zusammensetzung $\mathrm{P}_{4} \mathrm{O}_{6} \mathrm{~S}_{4}$ besitzt, oder mit anderen Worten ein Additionsprodukt von Phosphorigsäureanhydrid und Schwefel ist, das wurde durch Dampfdichtebestimmungen festgestellt. Diese wurden nach der Victor Mererschen Methode in einer Atmosphäre von reinem Stickstoff und bei einer 'Temperatur von $350^{\circ}-400^{\circ}$ unter Benützung eines Bades von geschmolzenem Blei ausgeführt. Eine Zersetzung war bei dieser Temperatur nicht zu bemerken; beim Abkühlen fand sich die unveränderte Substanz krystallisiert an den Wänden der cylindrischen Röhre vor.

Hierbei wurden die folgenden Resultate erhalten:

\begin{tabular}{crrrc} 
& \multicolumn{1}{c}{ I. } & \multicolumn{1}{c}{ II. } & \multicolumn{2}{c}{ III. Berechnet für } \\
Gewicht der Substanz & 0.2853 & 0.3558 & 0.2700 & \multicolumn{1}{c}{$\mathrm{P}_{4} \mathrm{O}_{6} \mathrm{~S}_{4}$} \\
Dichtigkeit, $\mathrm{H}=1$ & 180.4 & 171.9 & 170.8 & 174 \\
$\Rightarrow \quad$ Luft $=1$ & 12.5 & 11.9 & 11.8 & 12.1
\end{tabular}

Die Bestimmungen II und III wurden mit Krystallen ausgeführt, welche durch Sublimation des destillierten Oxysulfides im Vacuum erhalten worden waren.

Phosphoroxysulfid zerfliefst rasch an der Luft und riecht dann nach Schwefelwasserstoff. Es wird schnell von Wasser aufgelöst, indem es Schwefelwasserstoff und zuerst Metaphosphorsäure bildet.

$$
\mathrm{P}_{4} \mathrm{O}_{6} \mathrm{~S}_{4}+6 \mathrm{H}_{2} \mathrm{O}=4 \mathrm{HPO}_{3}+4 \mathrm{H}_{2} \mathrm{~S} \text {. }
$$

Die Metaphosphorsäure geht eventuell rasch in Orthophosphorsäure über, wenn die Lösung auf dem Wasserbade abgedampft wird.

Phosphoroxysulfid ist leicht löslich im doppelten Volumen von Schwefelkohlenstoff, aus welchem es unverändert auskrystallisiert. Es ist ebenfalls löslich in Benzin, auf welches es jedoch einwirkt, indem die Flüssigkeit dunkel wird und sich schwefelhaltige Produkte bilden.

Die isolierten, farblosen Krystalle von Phosphoroxysulfid, wie sie durch Sublimation im Vacuum erhalten werden, bestehen aus rektangulären Prismen, die häufig eine Länge von 2-3 Millimeter und eine Dicke von 1-2 Millimeter erreichen; gelegentlich wurden viel grölsere Krystalle gewonnen. Eine grofse Anzahl von Krystallen wurde goniometrisch untersucht und trotz ihres schnellen Zerfliefsens 
gemessen. Dieses Resultat wurde erhalten durch Anwendung der sinnreichen kleinen Vorrichtung, wie sie von Fuess in Berlin an dem grofsen Horizontal-Kreis-Goniometer angebracht ist. Der wesentliche Teil der Vorrichtung besteht aus einer kleinen geschlossenen Glaszelle, welche so konstruiert ist, dafs sie unten ein Trockenmittel aufnimmt, wie beispielsweise Chlorcalcium oder Phosphorsäureanhydrid, und dafs sie auf den justierbaren Tisch anstatt des gewöhnlichen Krystallhalters pafst. Der Krystall befindet sich während der Messung in dieser Trockenkammer, und der Lichstrahl geht vom Kollimator zum Krystall und vom Krystall gerade auf das Beobachtungsfernrohr durch zwei nahe anliegende Wandungen des Gefälses, welche aus Spiegelglas bestehen.

Bei der Untersuchung aller Krystalle wurden keine Flächen beobachtet, aufser denjenigen von rektangulären Prismen und der Basis, da alle die vielen Winkel, welche gemessen wurden, nahezu und in verschiedenen Fällen, genau $90^{\circ}$ waren. Prüft man die Krystalle im parallel polarisierten Licht, indem man durch eine der drei Flächenpaare sieht, so findet man, dafs die Krystalle immer isotrop sind, während die anderen Flächen parallel zu den Prismenkanten auslöschen. Im konvergierenden Licht sind die einachsig konzentrischen Ringe mit dem dunklen Kreuze durch die isotrope Fläche sichtbar, vorausgesetzt, dals der Krystall in dieser Richtung wenigstens zwei Millimeter dick ist; ist er dünner, so ist nur das Kreuz sichtbar, was von der schwachen, doppelten Brechung herrührt. Dreht man den Krystall, so erscheint das Kreuz feststehend und öfnet sich nicht zu einer Hyperbel, wenn es nicht falsch eingestellt ist. Die Krystalle erscheinen deshalb einachsig; sind sie dennoch zweiachsig, so müssen die Winkel zwischen den optischen Achsen sehr klein sein. Sie sind deshalb wahrscheinlich tetragonale Prismen, welche durch die Basis begrenzt sind.

Obgleich mehrere gesonderte Präparate dargestellt wurden, so konnten doch niemals andere Flächen beobachtet werden; alle Krystalle waren homogen und zeigten nur Prismenflächen und die Basis. Um, wenn möglich, Krystalle, welche andere Formen zeigen und die Bestimmung der Achsenverhältnisse ermöglichen, zu erhalten, wurde eine beträchtliche Quantität Oxysulfid, das man durch Sublimation im Vacuum erhalten hatte, in Schwefelkohlenstoff aufgelöst, und der letztere langsam im Vacuum von dem einen Glied eines Winkelrohres zum anderen verdampft, indem man das zweite Glied mit Eis abkühlte. 
Es wurden abermals grofse, wohlausgebildete Krystalle erhalten, aber auch diese bestanden, wie die durch Sublimation erhaltenen, aus tetragonalen Prismen, welche durch die Basis begrenzt waren und sonst keine anderen Flächen aufwiesen.

Wie oben erwähnt, setzt sich das Oxysulfid zuweilen als eine farblose, zähe Flüssigkeit an und zwar besonders in jenem Teil der Röhre, welcher der Oberfläche des Glycerinbades zunächst ist. Beim Abkühlen erstarrt diese Flüssigkeit zu einem durchsichtigen. Glase, welches bald früher, bald später zu Krystallen derselben Form wie die isoliert dargestellten entglast. Gewöhnlich geht die Bildung der Krystalle in ungefähr einer Stunde vor sich. In einem Fall jedoch erfolgte die Entglasung plötzlich einige Tage nach der Darstellung der Substanz, und es bildeten sich hierbei seltsam gefiederte Aggregate der tetragonalen Krystalle.

Die Thatsache, dars die isolierten Krystalle, die glasige Modifikation und die gefiederte Form identisch sind, wurde du ch direkte Analyse bewiesen. Die gefiederten Gebilde sind lediglich Aggregate von kleinen tetragonalen Prismen, und die Nadeln sind ebensolche Prismen, welche stark in der Richtung ${ }^{\bullet}$ der vertikalen Achse entwickelt sind.

Royal College of Sciences. S. Kensington-London. 